\title{
Drug users' perceptions, demands, and suggestions for non-occupational post-exposure prophylaxis (nPEP) services in China: a qualitative study
}

Kedi Jiao

Shandong University

Haochu Li

Shandong University

Dapeng Zhang

Blue City Holdings, Ltd

Zhenxia Jiang

Qingdao Municipal Center for Disease Control and Prevention

Yuxi Lin

Shandong University

Xueyuan Liu

Shandong University

Hengmin Xu

Shandong University

Xuemei Yan

Shandong University

Haoqing Tang

Shandong University

Wei Ma ( $\square$ weima@sdu.edu.cn )

https://orcid.org/0000-0002-8092-3280

Research article

Keywords: Drug user, HIV/AIDS, Non-occupational post-exposure prophylaxis, China, Qualitative

Posted Date: October 1st, 2019

DOI: https://doi.org/10.21203/rs.2.15524/v1

License: (1) (1) This work is licensed under a Creative Commons Attribution 4.0 International License.

Read Full License 


\section{Abstract}

Background Drug users are regarded as a high-risk population of HIV infection. Non-occupational postexposure prophylaxis (nPEP) is internationally regarded as an effective biomedical prevention against HIV but still a small-scale pilot project in China at present. The aim of this study was to understand drug users' perceptions demands and suggestions for nPEP service in China.

Methods Nineteen eligible drug users were recruited in this qualitative study. Semi-structured in-depth interviews were conducted by trained interviewers. All of the interviews were audio-taped, transcribed verbatim and analyzed using thematic framework analysis.

Results Most of participants had not heard of nPEP and none of them had used this service. After being informed basic knowledge of nPEP, about half of the participants reported willingness to use it. The facilitators included scare of HIV infection and regarding nPEP as a remedial measure in emergency. The barriers included low willingness for fatigue and lethargy after taking drugs, high price and side effects of nPEP medication, long course of nPEP treatment, and fear of privacy disclosure. Most of the participants thought the availability of nPEP would not increase the occurrence of unprotected intercourse and supported the routinization of nPEP services. Most participants chose social media for nPEP propaganda and pharmacies for providing nPEP.

Conclusions It is imperative to strengthen propaganda and education of nPEP among drug users, and to encourage them to proactively use nPEP after exposure. Price regulation, side effect management and privacy protection need to be managed well when nPEP is routinized.

\section{Background}

Illicit drug use is recognized as a contributor to the disease burden worldwide [1]. It was estimated in 2016 that more than a quarter of billion people worldwide had used illicit drugs at least once in the previous year [2]. The number of newly HIV-infected injection drug users increased from 114,000 in 2011 to 152,000 in 2015, and it was estimated in 2016 that the prevalence of HIV among injection drug users worldwide was $11.8 \%$ [2]. Special attention should also be paid to the prevalence of club drugs that have been gradually increasing in many parts of the world over the past two decades [3]. The term "club drugs" refers to a category of drugs with a connection to clubs, "raves" or dance parties (e.g., methamphetamine, ketamine, Magu pills and ecstasy) [4]. In China, club drugs are commonly called newtype drugs for the reason that they are relatively new compared to "traditional drugs" (e.g., heroin, marijuana) [5]. In this study, the drug users were defined as people who used "traditional drugs" and/or the new-type drugs by any means including injection.

Post-exposure prophylaxis (PEP) is the use of antiretroviral (ARV) drugs within 72 hours of exposure to HIV in order to prevent infection. PEP includes counseling, first aid care, HIV testing and 
administration of a 28-day course of ARV drugs with follow-up care [6]. PEP was first used for occupational exposure of HIV in the late 1980's, then gradually extended to non-occupational postexposure prophylaxis (nPEP) including after risky injection drug use and sexual contact [7]. The World Health Organization guidelines for PEP were first recommended in 2014 for all populations in both occupational and non-occupational exposures [8]. At present, nPEP service has been gradually routinized in many regions of the world. The guidelines of PEP in many countries (e.g., USA, UK and Belgian) have been issued for years and updated recently [9-11].

In China, needle sharing among injection drug users had declined dramatically in recent years with the efforts of national harm reduction programs, but the rapid emergence of new-type drugs brought a new challenge [12]. As of the end of 2018, there were about 2,404,000 drug users nationwide, decreasing by $5.8 \%$ year-on-year, and $56.1 \%$ of whom were methamphetamine abusers [13]. Studies have shown that the use of new-type drugs could increase the risk of HIV infection and transmission by encouraging highrisk sexual behaviors [14,15]. There were 849,602 reported cases of people living with HIV/AIDS (PLWH) in China by the third quarter of 2018 [16]. The percentage of new HIV infection through injection drug use decreased from $13 \%$ in 2012 to $2.4 \%$ in 2018, while the percentage of new infection through sexual transmission (including both homosexual transmission and heterosexual transmission) increased from 79.5\% in 2012 to $93.8 \%$ in 2018 [16,17]. PEP has been widely used for occupational protection against HIV, while nPEP is just piloted in a small scale in China. Up to date, few study concentrated on nPEP in China. A study in Guangxi Province reported that among 344 men who have sex with men (MSM), 22.1\% had heard of nPEP and 60.2\% had expressed demands for nPEP [18]. Another study in Jinan surveyed 171 men receiving nPEP found no HIV conversion due to nPEP [19].

Few studies focused on the subgroup of drug users to explore their views about nPEP before the routinization of this service. The goal of this study was therefore to understand drug users' perceptions, demands and suggestions for this specific nPEP service in China through qualitative in-depth interviews. This study will provide the insiders' view about nPEP use from the perspective of drug users, a key population for HIV prevention, and therefore facilitate the development of nPEP guideline and routinization of the services in China and worldwide.

\section{Methods}

The qualitative study was conducted in Qingdao and Shanghai, two major coastal cities of China in August 2018. In the past decade, drug trafficking and abusing were pretty common in Qingdao which is known as a distribution center for drugs from Japan, South Korea and Russia [20]. Similarly, club drugs abuse is prevalent in Shanghai, a municipality with developed entertainment industry. A study in Shanghai reported that $67.8 \%$ of 276 club drug users had unprotected sex [5]. In this study, convenience 
sampling was used to recruit 10 drug users through referral from local community-based organizations (CBOs) and local Centers for Disease Control and Prevention (CDC). Eligible participants should: (1) reach 18 years old or above; (2) live in the study area for at least 3 months; (3) have formerly taken or been taking illicit drugs; (4) be HIV-negative or unknown of HIV status; and (5) provide informed consent and voluntarily participate in the survey. The exclusion criteria included: (1) self-reported HIV-positive; (2) suffering from mental health diseases or conscious impairment.

In-depth interviews (IDI) were arranged in a private room in the local CDC or CBO. The interviews were conducted by trained interviewers using mandarin Chinese after the written informed consents were provided. The participants were assured of confidentiality, privacy protection (e.g., use of pseudonyms), and their rights to refuse to answer any questions or withdraw at any stage without prejudice. A semi-structured interview guide was developed according to a review of the literature, and collected information about drug use behaviors and related high-risk sexual behaviors, knowledge and experiences of nPEP, demands for nPEP and associated factors, potential impacts of nPEP on high-risk behaviors and perceptions of nPEP routinization. Each IDI lasted approximately 40-50 minutes. After completing the interview, each participant was compensated $200 \mathrm{RMB}$ for time spent and transportation costs.

All of the interviews were audio-taped, transcribed verbatim in Chinese and analyzed using thematic framework analysis [21]. The major steps of data analysis were as follows. First we read the transcripts carefully to gain a comprehensive understanding in order to list key ideas and recurrent themes related to perceptions, demands and suggestions for nPEP services. Next, a thematic framework that identified all the key issues, concepts, and themes by which the data can be examined and referenced was developed to guide the coding process. A thematic framework was developed according to interview purposes and interview guide, which mainly includes perceptions of nPEP (knowledge and experiences), demands for nPEP (facilitators and barriers) and suggestions for nPEP (routinization, institutions and price). Then this framework was applied to code every transcript using ATLAS.ti 5.0. Afterwards, by inductive reasoning, we identified participants' perceptions of nPEP, factors prohibiting and facilitating their demand for nPEP, and suggestions for nPEP services. Furthermore, accompanying quotes and related themes were clustered and synthesized. Finally, we translated the relevant quotes into English.

The study was approved by the Ethical Review Committee (ERC) of Public Health in Shandong University.

\section{Results}

\section{Socio-demographic characteristics}


A total of 19 eligible participants were recruited, including 10 in Shanghai and 9 in Qingdao. The socio-demographic characteristics of the participants were shown in Table 1. Among the participants, $42.1 \%$ were aged 30 and below, and $26.3 \%$ were aged 50 and above. Fourteen participants were males and 5 were females. Most of them (84.2\%) were employed with different monthly income. The majority of participants (63.2\%) had never married.

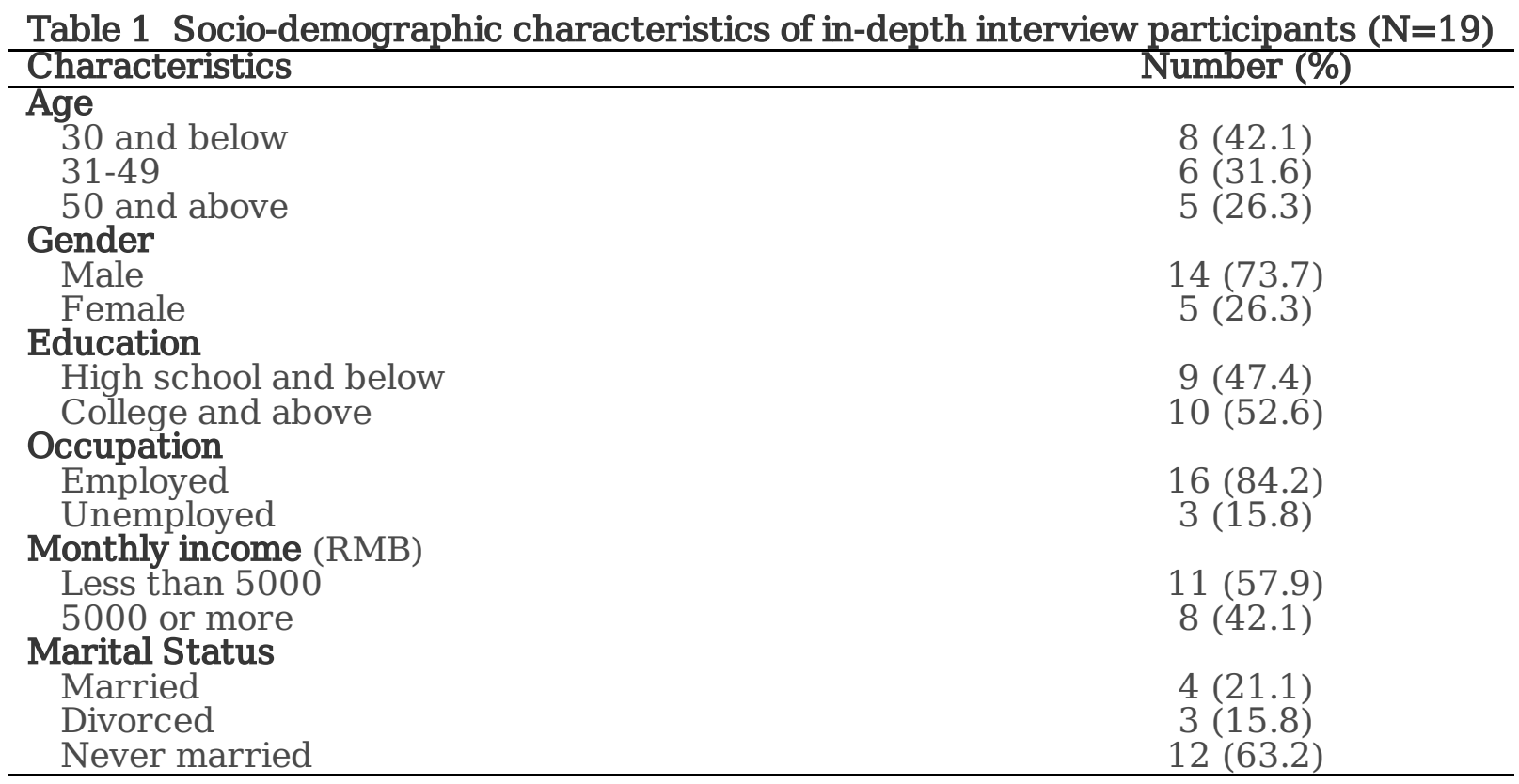

\section{Awareness and perceptions of nPEP services}

Most (17/19) participants reported never hearing of nPEP. Two participants had a rough look on the Internet or heard of it in the pharmacy. However, neither of them had clear knowledge about nPEP or how to get access to nPEP services. None of the participants had any experience of using nPEP.

"I have heard of it (nPEP) in the pharmacy. They said the medication was expensive. I did not try to know more about it." (27-year-old, QD09)

\section{Demands for nPEP service and associated factors}

After being briefly introduced the basic knowledge and effectiveness of nPEP in HIV prevention and intervention, half of the participants reported no demand for the nPEP services. Multiple barriers to using nPEP services were reported. In many cases, participants did not think of obtaining nPEP within the recommended time limit because they would be strung out on drugs for a few days. Besides, the nPEP medication was not always available in the local hospitals. The participants would have to get medication from other institutions in case of exposure, where could be too far to reach.

"I cannot remember such a thing (nPEP) within 72 hours at all. Someone just like me will choose to sleep for a day or one and a half days after taking drugs. Some others with violent temper are impossible to keep calm. The staying power of drugs is too strong for me to keep in mind in taking medications (nPEP). 
The medications are not available in the hospitals. I have to find institutions even if keeping conscious. I would not like to buy medications in this case." (24-year-old, QD05)

Second, some participants reported that they couldn't bear the cost of nPEP services, especially for those participants who relied on basic living allowance. Nevertheless, some participants claimed that they would seek nPEP at any cost if exposed to HIV.

"The biggest barrier (for using nPEP) is the high price which may not be accepted by most people. Only people with high-income may afford it. "(57-year-old, SH04)

"I can accept the price. I have to weigh the pros and cons. What should I do if I get AIDS? I may lose my job. I just spend money to protect myself." (27-year-old, QD09)

Third, side effects of nPEP might be another concern. Many participants worried about continued weight loss, headaches and other specific symptoms during treatment. They were reluctant to accept nPEP if the side effects are too severe.

"I worry about (the side effects). I spend a lot of money tidying up my appearance, so it's better to have no side effects such as falling out hair, skin rash and so on." (23-year-old, QD06)

"It's all right to avoid (unprotected sex) and insist on taking medicines for one month. I worry about the side effects of the medicine because I have a history of allergy. I am allergic to something and afraid of being reactive (to $n P E P$ medicines)." (24-year-old, QD01)

The fourth barrier is the difficulty of adherence to the whole course of nPEP. Some participants indicated that a 28-day course of nPEP was too long for them to keep compliance. For one thing, they had difficulties in persistently taking medications every day. For example, it was inconvenient for them to explain to friends and colleagues about why they take medications every day. For another, some participants thought that some peers may have unprotected sex again within the course of taking nPEP medications.

"We truly care about the side effects of nPEP medications. For example, the side effects of medicines for cold are significant such as marasmus, fatigue or brain symptoms. For instance, you will feel sleepy and fatigued after taking nPEP medications. It is reasonable to explain to friends as catching a cold but illogical to maintain this condition for 28 days, the impact of which is huge. For one thing it is on account of occupation, for another the colleagues will suspect." (24-year-old, QD05)

"Yes. It is extremely troublesome. Just think normally, I suppose people definitely (have sex) more than once in a month. However, it is very likely for people to have sex again during the course of taking $n P E P$ medications." (24-year-old, QD01)

Fifth, fear of privacy disclosure was another barrier of seeking nPEP. One participant explicitly pointed out that confidential information was easily leaked in the hospitals owing to case registration system, doctors' incautions and disordered environment of the hospitals. 
"But this sort of thing (AIDS) is disgraceful and confidential. For example, I worry about such a thing (AIDS) after sexual behavior and go to a hospital for checkup. If there are a lot of patients around, I will feel humiliated when the doctors don't hear me and asked me 'what' loudly. If I am tested HIV-positive and the doctors continually repeat, you will feel embarrassed. In this regard, a separated and private institution instead of a hospital is more suitable for this thing (nPEP)." (24-year-old, QD05)

Last, some participants reported that they had a regular sexual life and used condoms consistently during sexual intercourses, which justified their no demand for nPEP "I think every aspect of my life is stable, especially in sexual life. It seems that I have no demand of it (nPEP) now." (51-year-old, SH06)

For participants who reported willingness to use nPEP, facilitators included (1) perceiving serious consequences of HIV infection and (2) using nPEP as a remedial measure in emergency. "Maybe I need it (nPEP). The particular reason is that I am also afraid of getting AIDS. It sounds terrible if someone get AIDS." (57-year-old, SH04)

"(The nPEP service) is better than nothing. At least there is a way to remedy. It's not half bad to know that nPEP medicine can reduce the risk of HIV infection when I cannot control myself." (23-year-old, QD07)

\section{Potential impacts of nPEP service on high-risk sexual behaviors}

Most of participants reported the availability of nPEP services would not lead to an increase of their practices of unprotected sexual intercourse because they understood that nPEP medications may not absolutely protect them from getting HIV infection and there are potential side effects of the medications. Meanwhile, four participants thought their high-risk sexual behaviors might slightly increase with the initiation of nPEP services.

"I think I'll be careful. You can't tell immediately if someone has AIDS. It's too late to know a year or two later, so it seems better to be cautious beforehand. So I don't think (the nPEP service) is going to affect my sexual behavior, and it's going to be a protective measure." (23-year-old, QD07)

"I don't want to be more unscrupulous, but to indulge myself a little. For example, there used to be a lot of things I couldn't do, but now I'm wearing a suit of armor." (50-year-old, SH02)

\section{Suggestions for nPEP service}

Page $7 / 16$ 
Most (15/19) participants thought it was necessary to routinize nPEP services among drug users. Whereas two participants held the opposite attitudes who thought drug users never mind their own health condition.

"I definitely think the routinization of nPEP is necessary. At least the service will help me to prevent HIV infection ahead of time. I did not know the service in detail before." (27-year-old, QD09)

"There is no need to routinize (nPEP). Drug users care nothing if they have been taking drugs. Because they are clear that taking drugs is by no means a right thing, still they do so. They are indifferent to other things." (23-year-old, QD06)

For those who supported the routinization of nPEP, social media was cited as the most popular form of advocacy, followed by face-to-face outreach by healthcare professionals or local CBO community groups. Other forms of publicity include posters, bill boards and lectures.

"You can routinize through the WeChat. But don't organize group chat, just through Wechat applet. You can give a sample introduction on Internet, and let them (drug users) spread forwardly." (24-year-old, QD05)

With regard to the institutions providing nPEP services, 10 (52.6\%) participants preferred pharmacies, which guarantee both convenience and privacy protection. Four (21.1\%) participants selected hospitals for their authoritativeness and credibility. Also, there was a suggestion of initiating nPEP services on the Internet if possible.

"It is undoubtedly pharmacies (to get nPEP service) which are more convenient and secure. So is CDC. By comparison, it is not trustful to get medications from friends." (50-year-old, SHO3)

In Shanghai, only two (20\%) participants could afford nPEP medications. By contrast, six (66.7\%) participants could bear the current cost of nPEP in Qingdao and indicated that they attached more importance to the effect than the price. When asked the perception of cost of nPEP and affordable maximum price, most participants thought current price is a little high and gave their reasonable range, namely 1000 to 2000 RMB. There was a suggestion that the cost of medication should be shared in the form of allowance by the government.

"If so, I think the government should share half or a portion of cost. Then we bear the rest by ourselves." (30-year-old, QD03)

\section{Discussion}

This study explored the drug users' perceptions, demands and suggestions for nPEP services in China through qualitative in-depth interviews. We found participants' perceived barriers that were related to their no demand for nPEP services, their perceived facilitators, and their suggestions on routinization, institutions, price of nPEP services. 
Only two participants had heard of nPEP and none of them had used nPEP medications in this study, which indicated that nPEP had not been fully understood among drug users in China. Another study targeting MSM in China in 2017 found that 22.1\% of participants had heard of nPEP before their surveys [18]. By contrast, 48.5\% (272 of 500) of HIV-negative MSM had heard of nPEP in a Canadian study in 2016 [22].

Half of participants expressed no demand for nPEP after being informed of the basic knowledge of the nPEP services in this survey. Multiple barriers to using nPEP services were reported, including low willingness for fatigue and lethargy after taking drugs, high price and side effects of nPEP medications, long course of nPEP treatment, and fear of privacy disclosure. Participants pointed out that the feeling of fatigue and lethargy after taking drugs would last for a few days, lowering the willingness to get nPEP. The described symptoms were in accord with a research which showed that there were four periods after taking drugs, namely, excitement period (1-2 days or 3-5 days), fatigue period (1-3 days), melancholy period (1-3 days) and recovery period [23]. With effects of drugs descending or eliminating, drug uses in fatigue period may lose willingness to get nPEP service due to exhaustion. Consequently, warning education and behavioral intervention such as encouraging consistent condom use and avoiding multiple sex partners should continue to reinforce. Specifically, peer education had been proved an effect health education model and widely used among drug users in developing countries [24]. It may be feasible that choosing and training a small part of influential drug users in their circles on nPEP related knowledge to remind drug users of seeking nPEP in time after exposure. Besides, pre-exposure prophylaxis (PrEP) can be considered to initiate among drug users in the future in view of proposals of some participants that hoping to obtain medications before taking drugs when they were conscious.

The high cost of nPEP medications was also one of the deterrents for seeking nPEP services. In this study, half of participants in Shanghai whose monthly income less than 3000 RMB could not afford medication. It seemed that affordability of nPEP medications depended on income levels of participants to a certain extent. Different from this study, a study in the United States revealed that men whose annual individual income less than $\$ 30,000$ were more likely to use nPEP compared to those with high income. However, it was noteworthy that health insurance could often cover the costs of nPEP drug in US and their data also suggested that those with lower incomes might have difficulty in paying for nPEP without financial support [25]. It reminded us that we ought to take the income levels of drug users in different areas into account when drafting the price of nPEP. In general, the medication price should neither be too high or too low. Excessively high price may restrict the demand for nPEP as showed in our research. Conversely, if medication pricing is too low, it will have little economic constraints on their nPEP use, which may encourage excess high-risk sexual behavior. Just as a behavioral economic analysis of Internet 
use suggested, having free access to the Internet for young adults might encourage excessive, problematic use [26].

Some participants claimed that they were not likely to accept nPEP if adverse drug reactions were intolerable. In previous studies, adverse effects were always one of the important barriers of nPEP implement [27-30]. But in general, the side effects of ARV medication for most subjects within a short period were relatively moderate, mainly including headache and gastrointestinal symptoms. A ten-year retrospective analysis found that $56 \%$ of participants reported side effects starting nPEP, among them only 5\% interrupted treatment [31]. Most common side effects could be found during follow-up and improved through symptomatic treatment. The physicians should render detailed medication guidance while prescribing to alleviate patients' unwarranted fear about side effects of nPEP. Furthermore, an initial prescription for 3-5 days medication (commonly referred to as starter packs) with a follow-up visit could be alternatively chose to assess medication side effects and provide additional counseling [32].

The difficulty of adherence to the whole course of nPEP was another barrier. Some participants complained that 28-day course of treatment was too long to complete and felt inconvenient to take medicine every day. The problem of adherence was also demonstrated in several studies [29,33,34]. A study in Singapore found only $42 \%$ of 502 nPEP cases were adherent to nPEP [29]. One prospective observational study in Belgium recorded 66.4\% had a documented completion of 28-day course of nPEP among 1881 patients receiving nPEP [34]. Accordingly, the poor completion rates prevented patients from receiving optimal post-exposure care. Educations on medication adherence for patients need to be attached great importance. Besides, regimen simplification can improve inconvenience of taking medication to further raise compliance. A meta-analysis found that higher pill burden was associated with lower adherence rates and the average adherence was modestly higher in once-daily regimens than twice-daily regimens [35]. Kahn et al. proposed some suggestions that contributed to a higher adherence rate to $\mathrm{nPEP}$, including detailed explanations of the importance of adherence, professional mental health support and regimens with fewer medications [36].

In addition to the barriers above, concerns regarding privacy disclosure in the hospitals, which may bring about stigma and discrimination, were raised by most participants. HIV and AIDS-related stigma and discrimination were still common in healthcare settings [37-39]. A qualitative study in France reported that physicians' aggressive words in face-to-face interactions could bring up negative personal experiences for subjects with ongoing follow-up for HIV infection and PEP history [30]. Previous studies in China also suggested that HIV-related stigma and discrimination from healthcare providers could hinder the utilization of HIV prevention and treatment $[40,41]$. Addressing privacy disclosure and associated discrimination in healthcare settings is crucial to routinize nPEP. From the perspective of medical staff providing HIV/AIDS services, systematic training of HIV-related laws and regulations (e.g., Regulations 
on AIDS Prevention and Treatment in China) is imperative. From high-risk populations' aspect, essential psychological care and social support may reduce self-stigma and unwarranted fear.

Participants who reported demands for nPEP services were mostly due to perceiving serious consequences of HIV infection or regarding nPEP as a remedial measure in emergency. The subjects with high risk perceptions, namely those who realized that the exposures of high-risk sexual practices were more likely to result in HIV infection, inclined to turn to nPEP. Teo et al. found that self-perceived chances of infecting HIV and severity of the situation could facilitate nPEP treatment [29]. The new-type drugs can act on neurons and create feelings of pleasure as central nervous system stimulants [42]. After drug users take drugs, they can't control themselves and are usually associated with unprotected intercourses, inconsistent condom uses and multiple sexual partners. The nPEP service is regarded as an emergency medical response at this point.

The participants gave some suggestions on routinization, institutions and price of nPEP services in this study. Almost all the participants supported to routinize nPEP among drug users. But it was noteworthy that our finding addressed a social concern termed "sexual risk compensation" or "risk behavior disinhibition" which referred to phenomenon that the introduction of HIV prevention measures might introduce an overly optimism of protection among users and increase the risk behavior in other forms [43]. Although most participants in this study thought the initiation of nPEP would not lead to an increase of high-risk sexual behavior, we should remain cautious and further studies need to be done in China to justify the routinization. Social media (e.g., Wechat and microbolg) was cited as the most popular form of advocacy, followed by face-to-face propaganda. Social media is a novel approach and has demonstrated encouraging effects to improve HIV services for many key populations worldwide [44]. More than half of participants chose pharmacies as institutions providing nPEP services mainly for convenience and confidentiality. Considering that nPEP medication must be prescribed by clinicians and risk assessment with a series of biochemical examination needed to be done ahead of treatment, authoritative institutions (e.g., hospitals and CDC) should undertake major responsibilities of offering nPEP, and designated pharmacies cooperating with local hospitals could alternatively act as auxiliary institutions. The current price of nPEP is assumed to be a little high and advised to range from 1000 to 2000 RMB. It may be feasible to include nPEP medicines into medical insurance reimbursement in the future. In Switzerland, the cost of nPEP is charged directly to patients and then partially reimbursed through medical insurance [31].

There are several limitations in this study. First of all, only a small sample of drug users was included in this study and they were limited to referral from local CDC and CBOs. Consequently, our conclusions could not be generalized to the entire population like other exploratory studies. Second, we could not exclude the possibility that the participants deliberately concealed or inaccurately recalled their behavior 
information, which may lead to "information bias". Third, some participants in Shanghai had got off the drugs for several years, which might influence the willingness of turning to nPEP to a certain extent.

\section{Conclusions}

In order to reduce HIV infection in high-risk populations, it is imperative to strengthen propaganda and education of nPEP among drug users, and to encourage proactive use of nPEP after exposure. Price regulation, side effect management and privacy protection need to be managed well when nPEP is routinized. In addition, a guideline for nPEP use should be developed in China as soon as possible so that physicians have a standard reference while prescribing.

\section{Declarations}

\section{Ethics approval and consent to participate}

The written informed consents were provided before interview. The participants were assured of confidentiality, privacy protection (e.g., use of pseudonyms), and their rights to refuse to answer any questions or withdraw at any stage without prejudice during the interviews. Our study was approved by the Ethical Review Committee (ERC) of School of Public Health in Shandong University (20180904).

\section{Consent for publication}

Not applicable.

\section{Availability of data and materials}

The datasets used and/or analysed during the current study are available from the corresponding author on reasonable request.

\section{Competing interests}

The authors declare that they have no competing interests

\section{Funding}

The study was supported by the National Key Research and Development Program of China (2017YFE0103800) and the National Science and Technology Major Project (2018ZX10721102-003004).

\section{Authors' contributions}

WM conceived and designed the study. KJ, DZ, ZJ performed the experiments. KJ, YL, XL, HX, XY, HT transcribed and analyzed the data. KJ wrote the manuscript. HL and WM made critical revision of the manuscript. All authors read and approved the final manuscript. 


\section{Acknowledgements}

Acknowledgements should be made to local CDCs in study sites, Dr. Canrui Jin and Liyan Wang in People's Medical Publishing House for organizing the interviews.

\section{Abbreviations}

AIDS: Acquired Immune Deficiency Syndrome

ARV: antiretroviral

CDC: Centers for Disease Control and Prevention

CBO: community-based organizations

HIV: human immunodeficiency virus

IDI: In-depth interview

MSM: men who have sex with men

nPEP: non-occupational post-exposure prophylaxis

PEP: post-exposure prophylaxis

PrEP: pre-exposure prophylaxis

PLWH: people living with HIV/AIDS

\section{References}

1. Degenhardt L, Hall W. Extent of illicit drug use and dependence, and their contribution to the global burden of disease. The Lancet. 2012; 379:55-70.

2. $\quad$ United Nations Office on Drugs and Crime. World drug report, 2018.

http://www.unodc.org/wdr2018/prelaunch/WDR18_Booklet_2_GLOBAL.pdf. Accessed 15 Jun 2019.

3. Degenhardt L, Copeland J, Dillon P. Recent trends in the use of "club drugs": an Australian review. SUBST USE MISUSE. 2005; 40:1241-1256.

4. Chen X, Li X, Zheng J, Zhao J, He J, Zhang G, et al. Club Drugs and HIV/STD Infection: An Exploratory Analysis among Men Who Have Sex with Men in Changsha, China. PLOS ONE. 2015; 10:e126320.

5. Ding Y, He N, Zhu W, Detels R. Sexual Risk Behaviors among Club Drug Users in Shanghai, China: Prevalence and Correlates. AIDS BEHAV. 2013; 17:2439-2449.

6. WHO. HIV/AIDS [EB/OL].

https://www.who.int/news-room/fact-sheets /detail/hiv-aids. Accessed 30 Jul 2019. 
7. Beekmann SE, Henderson DK. Prevention of Human Immunodeficiency Virus and AIDS. INFECT DIS CLIN N AM. 2014; 28:601-613.

8. Beanland RL, Irvine CM, Green K. End Users' Views and Preferences on Prescribing and Taking Postexposure Prophylaxis for Prevention of HIV: Methods to Support World Health Organization Guideline Development. CLIN INFECT DIS. 2015; 60:S191-S195.

9. Goldschmidt RH. CDC Releases Updated Guidelines for Postexposure Prophylaxis After Sexual, Injection Drug, or Other Nonoccupational Exposures to HIV. AM FAM PHYSICIAN. 2016; 94:392-393.

10. Libois A, Florence E, Derdelinckx I, Yombi J.C, Henrard S, Uurlings F, et al. Belgian guidelines for nonoccupational HIV post-exposure prophylaxis 2017. ACTA CLIN BELG. 2018; 73:275-280.

11. Bamford A, Tudor-Williams G, Foster C. Post-exposure prophylaxis guidelines for children and adolescents potentially exposed to HIV. ARCH DIS CHILD. 2016; 102:78-83.

12. Wang L, Guo W, Li D, Ding Z, McGoogan J.M, Wang N, et al. HIV epidemic among drug users in China: 1995-2011. ADDICTION. 2015; 110:20-28.

13. General Office of National Narcotics Control Commission. Annual report in drug control in China, 2018. http://www.nncc626.com/2019-06/17/c_1210161797.htm. Accessed 30 Jul 2019. (In Chinese)

14. Hoenigl M, Chaillon A, Moore DJ, Morris S.R., Smith D.M., Little S.J. Clear Links Between Starting Methamphetamine and Increasing Sexual Risk Behavior: A Cohort Study Among Men Who Have Sex With Men. J Acquir Immune Defic Syndr. 2016; 71:551-557.

15. Melendez-Torres GJ, Bourne A. Illicit drug use and its association with sexual risk behaviour among MSM: more questions than answers? CURR OPIN INFECT DIS. 2016; 29:58-63.

16. National Center for AIDS/STD Control and Prevention, China CDC. Update on the AIDS/STD epidemic in China the third quarter of 2018. Chinese Journal of AIDS \& STD. 2018; 24:1075. (In Chinese)

17. National Center for AIDS/STD Control and Prevention, China CDC. Update on the AIDS/STD epidemic in China and main response in control and prevention in the third quarter of 2012. Chinese Journal of AIDS \& STD 2012;18:719. (In Chinese)

18. Zeng Z, Liu H, Xu J, Lan G, Wang L, Yin W. Demand for non-occupational post-exposure prophylaxis and its influencing factors among 344 men who have sex with men in Guangxi. Chinese Journal of AIDS \& STD. 2017; 23:620-624. (In Chinese)

19. Wang C, Duan Q, Chen Q, Bi Y, Li Y, Kang D. High-risk sexual behavior and preventive effects of men receiving HIV non-occupational post-exposure prophylaxis in Jinan. Chinese Journal of AIDS \& STD. 2018; 24:1221-1223. (In Chinese)

20. Jiang Z, Xiu C, Yang J, Zhang X, Liu M, Chen X. HIV test uptake and related factors amongst heterosexual drug users in Shandong province, China. PLOS ONE. 2018;13:e204489. 
21. Pope C, Ziebland S, Mays N. Qualitative research in health care. Analysing qualitative data. BMJ. 2000;320:114-116.

22. Lin SY, Lachowsky NJ, Hull M, Rich A, Cui Z, Sereda P, et al. Awareness and use of nonoccupational post-exposure prophylaxis among men who have sex with men in Vancouver, Canada. HIV MED. 2016;17:662673.

23. Han Q, Han D. A survey of depression status for neotype drug abuseres during compulsory detoxification. JOURNAL OF CLINICAL PSYCHOSOMATIC DISEASES. 2009;15:106-108. (In Chinese)

24. Medley A, Kennedy C, O'Reilly K, Sweat M. Effectiveness of Peer Education Interventions for HIV Prevention in Developing Countries: A Systematic Review and Meta-Analysis. AIDS EDUC PREV. 2009; 21:181206.

25. Mitchell JW, Sophus AI, Petroll AE. HIV-Negative Partnered Men's Willingness to Use Non-Occupational Post-Exposure Prophylaxis and Associated Factors in a U.S. Sample of HIV-Negative and HIV-Discordant Male Couples. LGBT HEALTH. 2016; 3:146-152.

26. Broadbent J, Dakki MA. How Much Is Too Much to Pay for Internet Access? A Behavioral Economic Analysis of Internet Use. Cyberpsychology, Behavior, and Social Networking. 2015; 18:457-461.

27. Gulholm T, Jamani S, Poynten IM, Templeton DJ. Non-occupational HIV post-exposure prophylaxis at a Sydney metropolitan sexual health clinic. SEX HEALTH. 2013; 10:438-441.

28. Li H, Cheng Y, Yang C. Safety, tolerability and effectiveness of HIV non-occupational prophylaxis in Taiwan. J INT AIDS SOC. 2014; 17:19736.

29. Teo AKJ, Tai BC, Chio MT, La HH. A mixed methods study of non-occupational post-exposure prophylaxis at an STI clinic in Singapore: Five-year retrospective analysis and providers' perspectives. PLOS ONE. 2018; 13:e202267.

30. Palich R, Martin-Blondel G, Cuzin L, Le Talec J, Boyer P, Massip P, et al. Experiences of HIV postexposure prophylaxis (PEP) among highly exposed men who have sex with men (MSM). SEX TRANSM INFECT. 2017; 93:493-498.

31. Tissot F, Erard V, Dang T, Cavassini M. Nonoccupational HIV post-exposure prophylaxis: a 10-year retrospective analysis. HIV MED. 2010; 11:584-592.

32. Smith DK, Grohskopf LA, Black RJ, Auerbach J.D, Veronese F, Struble K.A, et al. Antiretroviral postexposure prophylaxis after sexual, injection-drug use, or other nonoccupational exposure to HIV in the United States: recommendations from the U.S. Department of Health and Human Services. MMWR Recomm Rep. 2005; 54:1-20.

33. Minas B, Laing S, Jordan H, Mak DB. Improved awareness and appropriate use of non-occupational post-exposure prophylaxis (nPEP) for HIV prevention following a multi-modal communication strategy. BMC PUBLIC HEALTH. 2012; 12:906. 
34. Malinverni S, Gennotte A, Schuster M, De Wit S, Mols P, Libois A. Adherence to HIV post-exposure prophylaxis: A multivariate regression analysis of a 5 years prospective cohort. J INFECTION. 2018; 76:78-85. 35. Nachega JB, Parienti J, Uthman OA, Gross R, Dowdy D.W, Sax P.E, et al. Lower Pill Burden and OnceDaily Antiretroviral Treatment Regimens for HIV Infection: A Meta-Analysis of Randomized Controlled Trials. CLIN INFECT DIS. 2014; 58:1297-1307.

36. Kahn JO, Martin JN, Roland ME, Bamberger J.D, Chesney M, Chambers D, et al. Feasibility of postexposure prophylaxis (PEP) against human immunodeficiency virus infection after sexual or injection drug use exposure: the San Francisco PEP Study. J INFECT DIS. 2001; 183:707-714.

37. Wodajo BS, Thupayagale-Tshweneagae G, Akpor OA. HIV and AIDS-related stigma and discrimination in two referral hospitals in Ethiopia. Afr J AIDS Res. 2017; 16:137-144.

38. Kabbash IA, Abo AE, Elgendy MM, Abdrabo M.M, Salem H.M, Gouda M.R, et al. HIV/AIDS-related stigma and discrimination among health care workers at Tanta University Hospitals, Egypt. Environ Sci Pollut Res Int. 2018; 25:30755-30762.

39. Dong X, Yang J, Peng L, Pang M, Zhang J, Zhang Z, et al. HIV-related stigma and discrimination amongst healthcare providers in Guangzhou, China. BMC PUBLIC HEALTH. 2018; 18.

40. Liao M, Kang D, Tao X, Bouey J.H, Aliyu M.H, Qian Y, et al. Alcohol Use, Stigmatizing/Discriminatory Attitudes, and HIV High-Risk Sexual Behaviors among Men Who Have Sex with Men in China. BIOMED RES INT. $2014 ; 2014: 1-8$

41. Li X, Lu H, Ma X, Sun Y, He X, Li C, et al. HIV/AIDS-related stigmatizing and discriminatory attitudes and recent HIV testing among men who have sex with men in Beijing. AIDS BEHAV. 2012; 16:499-507.

42. Epple R, Blanes L, Beavis A, Roux C, Doble P. Analysis of amphetamine-type substances by capillary zone electrophoresis using capacitively coupled contactless conductivity detection. ELECTROPHORESIS. 2010; 31:2608-2613.

43. Cassell MM, Halperin DT, Shelton JD, Stanton D. Risk compensation: the Achilles' heel of innovations in HIV prevention? BMJ. 2006; 332:605-607.

44. Cao B, Gupta S, Wang J, Hightow-Weidman L.B, Muessig K.E, Tang W, et al. Social Media Interventions to Promote HIV Testing, Linkage, Adherence, and Retention: Systematic Review and Meta-Analysis. J MED INTERNET RES. 2017; 19:e394. 